\title{
Multiwavelength study of the TeV blazar Mrk 421 during a giant flare
}

\author{
A. Shukla ${ }^{1}$, V. R. Chitnis ${ }^{2}$, P. R. Vishwanath ${ }^{1}$, B. S. Acharya ${ }^{2}$, G. C. Anupama ${ }^{1}$, P. Bhattacharjee ${ }^{3}$, R. J. Britto ${ }^{2,3}$, \\ T. P. Prabhu ${ }^{1}$, L. Saha ${ }^{3}$, and B. B. Singh ${ }^{2}$ \\ ${ }^{1}$ Indian Institute of Astrophysics, II Block, Koramangala, 560034 Bangalore, India \\ e-mail: amit@iiap.res.in \\ 2 Tata Institute of Fundamental Research, Homi Bhabha Road, Colaba, 400005 Mumbai, India \\ 3 Saha Institute of Nuclear Physics, 1/AF, Bidhannagar, 700064 Kolkata, India
}

Received 3 December 2011 / Accepted 14 March 2012

ABSTRACT

\begin{abstract}
Aims. The nearby $(z=0.031) \mathrm{TeV}$ blazar Mrk 421 was reported to be in a high state of flux activity since November, 2009. We aim to investigate possible changes in the physical parameters of Mrk 421 during its high state of activity using multiwavelength data. Methods. We have observed this source in the bright state using the High Altitude GAmma Ray (HAGAR) telescope array at energies above $250 \mathrm{GeV}$ during February 13-19, 2010. Optical, X-ray and $\gamma$-ray archival data were also used to obtain the spectral energy distribution and light curves.

Results. Mrk 421 was found to undergo one of its brightest flaring episodes on February 17, 2010 by various observations in X-rays and $\gamma$-rays. HAGAR observations during February 13-19, 2010 at energies above $250 \mathrm{GeV}$ show an enhancement in the flux level, with a maximum flux of $\sim 7$ Crab units being detected on February 17, 2010. We present the spectral energy distributions during this flaring episode and investigate the correlation of the variability in X-ray and $\gamma$-ray bands.

Conclusions. Our multiwavelength study suggests that the flare detected during February 16 and 17, 2010 may have been caused by a passing shock in the jet.
\end{abstract}

Key words. astroparticle physics - BL Lacertae objects: individual: Mrk 421 - telescopes - gamma rays: galaxies

\section{Introduction}

Blazars are a subclass of active galactic nuclei (AGN) that are characterized by nonthermal emission extending from radio to high energies. The broadband radiation originates within a relativistic jet that is oriented very close to the line of sight. Spectral energy distributions (SEDs) of high-energy peaked TeV blazars show two broad peaks. The first peak is located between infrared to X-ray energies and the second peak at $\gamma$-ray energies. It is believed that the first peak of the SED is caused by synchrotron radiation by relativistic electrons gyrating in the magnetic field of the jet. The origin of the high-energy GeV/TeV peak is still under debate. This high-energy peak might be caused either by interaction of electrons with the photon field via inverse Compton (IC) scattering as in leptonic models or by interactions of protons with matter, magnetic field, or photon fields in hadronic models. The seed photon field for IC scattering in leptonic models may come from synchrotron emission by the same population of electrons that produce the low-energy bump (for a recent review of observations and models, see Krawczynski 2004) in synchrotron self-Compton (SSC) models and from the external photons from accretion disk (Dermer \& Schlickeiser 1993) or broad line region (BLR) (Ghisellini \& Madau 1996) in external Compton models. The hadronic models suggest that the high-energy peak might be caused by extremely energetic protons gyrating in a strong magnetic field, which emit synchrotron radiation (Aharonian 2000; Mücke et al. 2003), or as IC and synchrotron emission from a proton-induced cascade (Mannheim 1998).
The blazar Mrk $421(z=0.031)$ is the first extragalactic source to be detected at $\gamma$-ray energy $E>500 \mathrm{GeV}$ (Punch et al. 1992). Since the first detection with the Whipple Observatory $\gamma$-ray telescope in 1992, Mrk 421 has been detected with various other atmospheric Cherenkov telescopes (ACT) and air shower experiments (Petry et al. 1996; Zweerink et al. 1997; Aharonian et al. 1999; Bose et al. 2007; Yadav et al. 2007; Aleksić et al. 2010; Di Sciascio \& ARGO-YBJ Collaboration 2010), and its $\gamma$-ray flux has been found to be highly variable.

Mrk 421 was reported to be in a high state of activity during November 2009 to April 2010, with flaring behavior in X-ray (Isobe et al. 2010) and $\gamma$-ray bands detected in February, 2010. One of the brightest flaring episodes of this source was observed by various experiments on February 17, 2010. Preliminary results from different experiments show rapid flux variation in the very high energy (VHE) $\gamma$-rays from minute to hour time scales. During this bright outburst, the maximum VHE $\gamma$-ray flux $(>100 \mathrm{GeV})$ reached above 9 Crab units (Ong 2010), with an average flux of 4 Crab units.

Using the newly commissioned High Altitude GAmma Ray (HAGAR) telescope system, we observed Mrk 421 in its high state of activity during February to April, 2010 and also detected a very bright flare above $250 \mathrm{GeV}$.

In this paper, we study the multiwavelength behavior of Mrk 421 during its high state of activity in February, 2010 and follow the evolution of its SEDs over a period of seven days, based on data from HAGAR, Fermi-LAT, RXTE-PCA and Swift-XRT. We also present a brief introduction to the HAGAR 
telescope and the data analysis techniques used to detect pointlike $\gamma$-ray sources with HAGAR.

\section{HAGAR}

HAGAR, an array of ACTs using the wave-front sampling technique, is located at the Indian Astronomical Observatory (IAO), Hanle $\left(32^{\circ} 46^{\prime} 46^{\prime \prime} \mathrm{N}, 78^{\circ} 58^{\prime} 35^{\prime \prime} \mathrm{E}\right)$, in the Ladakh region of India, at an altitude of $4270 \mathrm{~m}$. The main motivation behind setting up the $\gamma$-ray array at a high altitude is to exploit the higher Cherenkov photon density and thus achieve a lower energy threshold (Cowsik et al. 2001). HAGAR consists of an array of seven telescopes in the form of a hexagon, with one telescope at the center. All seven telescopes have seven para-axially mounted front coated parabolic mirrors of diameter $0.9 \mathrm{~m}$, with a UV-sensitive photo-tube at the focus of individual mirrors. Each telescope is separated by $50 \mathrm{~m}$ distance from its neighboring telescope. The seven PMT pulses of a telescope are linearly added to form a telescope output called the Royal sum pulse. A coincidence of at least four Royal sum pulses out of seven, above a predetermined threshold, is taken within a time window of $150 \mathrm{~ns}$ or $300 \mathrm{~ns}$ depending on the zenith angle of the pointing direction to generate a trigger for initiating data recording. The HAGAR data acquisition (DAQ) system is CAMAC-based. Data recorded for each event consist of

a. Relative arrival time of the Cherenkov shower front at each mirror, as measured by TDCs with a resolution of $250 \mathrm{ps}$.

b. A real time clock (RTC) module synchronized with GPS is used to record the absolute arrival time of these events accurate up to $\mu$ s.

c. The density of Cherenkov photons at each telescope is measured by the total charge present in PMT pulses (this is recorded by using 12 bit QDCs).

d. Information on Royal sum pulses are recorded in the same way as the individual PMT pulses.

e. Latch information to indicate the triggered telescopes and other house-keeping information on various scalar readings.

In addition, a parallel DAQ using commercial waveform digitizers with a sampling rate of $1 \mathrm{GS} / \mathrm{s}$ (ACQIRIS make model DC271A) is also used. The direction and energy of $\gamma$-rays are estimated by measuring the relative time delays and densities of Cherenkov photons at each telescope respectively.

The performance of the HAGAR array has been studied by simulations, which were performed in two steps: (1) Cherenkov emission caused by $\gamma$-ray and cosmic-ray-induced air showers in the atmosphere, by using the Monte Carlo simulation package CORSIKA, developed by the KASCADE group (Heck et al. 1998); (2) study of the response of the array toward the Cherenkov radiation produced by the simulated showers. The performance parameters such as energy threshold, collection area and sensitivity of the experiment are obtained by a detector simulation package indigenously developed by the HAGAR collaboration. The energy threshold of the HAGAR telescope is estimated to be $204 \mathrm{GeV}$ for vertically incident $\gamma$-ray showers for $\mathrm{a} \geq$ four-fold trigger condition, for which the corresponding collection area is $3.2 \times 10^{8} \mathrm{~cm}^{2}$. HAGAR sensitivity is such that it will detect a Crab-nebula-like source at a significance level of $5 \sigma$ in $15 \mathrm{~h}$ of observation (Saha et al. 2011).

Cherenkov emission caused by induced air showers forms a spherical wavefront with a large radius of curvature and thickness of $\sim 1 \mathrm{~m}$ at the observation level. This Cherenkov emission mainly originates at the shower maximum region, which



Fig. 1. Typical space angle distribution plot obtained by HAGAR for a seven-fold trigger.

is at a height of about $5 \mathrm{~km}$ above the ground level at Hanle. This spherical wavefront is approximated as a plane wavefront in the data analysis procedure, which is a good approximation at the observation level. The arrival direction of each shower in the Cherenkov light pool is computed by measuring the relative arrival times of the shower front at different telescopes. The normal to this plane front gives the arrival direction of the incident shower. The angle between the direction of the shower axis and the pointing direction of the telescope is defined as the space angle $(\psi)$. This is estimated for every event by measuring the relative arrival time of the shower front at each telescope.

The observations were carried out by pointing all seven telescopes toward the source or background direction at a time. Each source run was followed (or preceded) by a background run with the same exposure time (typically $40 \mathrm{~min}$ ) and covered the same zenith angle range as that of the source to ensure that observations were carried out at almost the same energy threshold. Data selection was made by using parameters that characterize good quality data to reduce systematic errors.

Extraction of the $\gamma$-ray signal was carried out by comparing the ON source and OFF source space angle distributions obtained during the same night. The excess events were computed from $0^{\circ}$ to lower limit (LL), where LL is defined as the foot of distant half-maximum point computed by fitting a Gaussian function to the space angle distribution. Background space angle distribution was normalized with source space angle distribution by comparing the tails of the distributions (LL to $6.5^{\circ}$ ), since no $\gamma$-ray events are expected in this region. This normalization is required to compensate for possible changes in observation conditions or sky conditions during ON source and OFF source regions. Thus the number of $\gamma$-ray events are estimated as

Number of $\gamma-$ rays $=\Sigma_{0}^{L L}\left(S_{i}-c_{k} B_{i}\right)$,

where $S_{i}$ and $B_{i}$ are source and background events, respectively, and $c_{k}$ is the normalization constant obtained using the tail of the space angle distribution.

A typical space angle plot obtained for the seven-fold trigger is shown in Fig. 1. The dotted (black) line histogram corresponds to source observations, the dashed (green) line histogram corresponds to background observations. The histogram shown as solid (blue) line corresponds to the estimation of $\gamma$-ray events computed as the excess of ON source events over the normalized OFF source background events. 


\section{Multiwavelength observations and analysis}

Data obtained on Mrk 421 by HAGAR during its high state in 2010 were combined with archival data from Fermi-LAT, RXTE-ASM, Swift-BAT, Swift-XRT, RXTE-PCA, SPOL, and OVRO for a multiwavelength study. Details of the observations and analysis procedure used for each data set are discussed in this section.

\subsection{HAGAR}

The HAGAR observations of Mrk 421 were carried out for three months on moonless nights during February to April, 2010 at a mean zenith angle of $6^{\circ}$. A total of $30 \mathrm{~h}$ of data were collected during these observations. Suitable cuts were applied to the difference in the trigger rates of ON source and OFF source regions to ensure that observations were carried out at almost the same energy threshold. Some data were also rejected due to unavailability of all seven telescopes at the time of observations. After imposing these data quality cuts, a total of $18 \mathrm{~h}$ of observation was used for further analysis. Data were analyzed according to the procedure discussed in Sect. 2 and Bose et al. (2007). We analyzed only events with signals in at least five telescopes ( $\geq$ five-fold) to reduce systematic errors in our data. The $\geq$ fivefold events correspond to an energy threshold of about $250 \mathrm{GeV}$.

\subsection{Fermi-LAT}

The Large Area Telescope (LAT) is a pair production telescope (Atwood et al. 2009) onboard the Fermi spacecraft. It covers the energy range from $20 \mathrm{MeV}$ to more than $300 \mathrm{GeV}$ with a field of view $\geq 2.5$ sr. Fermi-LAT data ${ }^{1}$ of Mrk 421 obtained during February 12-22, 2010 above $100 \mathrm{MeV}$ were analyzed using the standard analysis procedure (ScienceTools) provided by the Fermi-LAT collaboration. A circular region of $10^{\circ}$ radius "region of interest (ROI)" was chosen around Mrk 421 for event reconstruction from the so-called "diffuse" event class data which has the maximum probability of being the source photons. We only retained events having a zenith angle $<105^{\circ}$ to avoid the background from Earth albedo. The spectral analysis on the resulting data set was carried out by including galactic diffuse emission component model (gll_iem_v02.fit) and an isotropic background component model (isotropic_iem_v02) with post-launch instrumental response function P6V3 DIFFUSE, by using unbinned maximum likelihood analysis (Cash 1979; Mattox et al. 1996). A power law spectrum was used to model the source spectrum above $100 \mathrm{MeV}$, with integral flux and photon index as free parameters. The flux, spectrum, and source location were determined by using unbinned GTLIKE algorithm.

\subsection{X-ray data from RXTE and Swift}

The Proportional Counters Array (PCA) (Bradt et al. 1993) is an array of five identical xenon-filled proportional counter units (PCUs). The PCUs cover energy range from $2-60 \mathrm{keV}$ with a total collecting area of $6500 \mathrm{~cm}^{2}$. The archival X-ray data from PCA onboard RXTE during February 13-19, 2010 was analyzed to obtain the X-ray spectrum and light curve. We have analyzed standard 2 PCA data that have a time resolution of $16 \mathrm{~s}$ with energy information in 128 channels. Data analysis was performed using FTOOLS (version 5.3.1) distributed as a part of HEASOFT (version 5.3). Data were filtered using the

\footnotetext{
${ }^{1}$ http://fermi.gsfc.nasa.gov/
}

standard procedure given in the RXTE Cook Book 4 for each of the observations. The background models were generated with the tool "pcabackest", based on RXTE GOF calibration files for a "bright" source (more than $40 \mathrm{ct} / \mathrm{s} / \mathrm{PCU}$ ). The PCA spectrum in the the energy range of 3-30 keV was fitted by using XSPEC with a cutoff powerlaw with line-of-sight absorption. The lineof-sight absorption was fixed to the neutral hydrogen column density at $1.38 \times 10^{20} \mathrm{~cm}^{-2}$ (Dickey \& Lockman 1990).

The XRT onboard Swift (Burrows et al. 2005) uses a grazing incidence Wolter I telescope to focus X-rays onto a CCD. The instrument has an effective area of $110 \mathrm{~cm}^{2}, 23.6$ arcmin FOV, 15 arcsec resolution (half-power diameter), and an energy range of $0.2-10 \mathrm{keV}$. The windowed timing (WT) mode data were used to obtain the spectrum $(0.3-3 \mathrm{keV})$ from Swift-XRT during February 13-19, 2010. Source photons were extracted using a box region with the length of 40 pixels and width about 20 pixels. Events with grades 0-2 were selected for the WT mode data. The spectral data were rebinned by GRPPHA 3.0.0 with 20 photons per bin. Standard auxiliary response files and response matrices were used. Spectra for this source were fitted using XSPEC version 12.3.1 with a model consisting of absorbed power law over the energy range of $0.3-10 \mathrm{keV}$.

The "Dwell" data from RXTE-ASM were obtained from the ASM website ${ }^{2}$ and were analyzed with the method discussed in Chitnis et al. (2009). A daily average flux between $15-50 \mathrm{keV}$ from Swift-BAT was obtained from BAT website ${ }^{3}$.

\subsection{Optical and radio data}

The optical and radio data made available in the Fermi multiwavelength support program websites ${ }^{4,5}$ are used in this study.

The optical observations were made using the SPOL CCD Imaging/Spectropolarimeter at Steward Observatory (Smith et al. 2009). The optical $V$-band photometric data were obtained.

The $15 \mathrm{Ghz}$ radio observations were made by using a $40 \mathrm{~m}$ single-dish telescope at Owens Valley Radio Observatory (OVRO). Details of the analysis are described in Richards et al. (2011).

\section{Results}

Mrk 421 was found to be in a high state of activity during the entire period of the HAGAR observations during February-April, 2010, and was in its brightest state in February, 2010. The $\gamma$-ray and X-ray fluxes decreased in the later months, but were still higher than those during the quiescent state. Results of observations of Mrk 421 using HAGAR during the three months period are given in Table 1 .

Figure 2 contains the daily light curve of Mrk 421 during February-April, 2010. The upper panel shows the daily average of VHE $\gamma$-ray flux obtained from HAGAR. The bottom panel shows the daily average from ASM onboard RXTE in $1.5-12 \mathrm{keV}$. It is clearly seen in the HAGAR as well as RXTEASM light curves that Mrk 421 was in its brightest state in February, 2010 in both VHE $\gamma$-rays and X-rays.

HAGAR telescope detected Mrk 421 in a high state of VHE $\gamma$-ray flux, during the period February 13-19, 2010. One of the

\footnotetext{
2 http://xte.mit.edu/

${ }^{3}$ http://heasarc .nasa.gov/docs/swift/results/

transients/

${ }^{4}$ http://james.as.arizona.edu/ psmith/Fermi/

5 http://www. astro. caltech.edu/ovroblazars/index.php? page $=$ home
} 


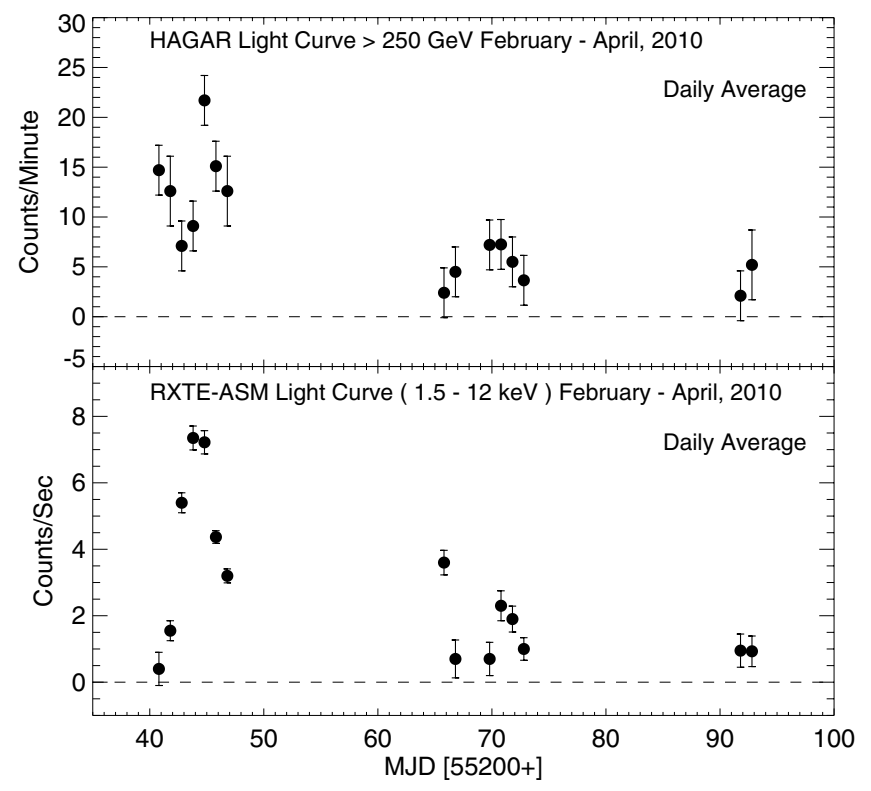

Fig. 2. Top panel: the daily average light curve of Mrk 421 during the period February-April, 2010 in VHE $\gamma$-rays above $250 \mathrm{GeV}$ from HAGAR and the bottom panel shows the X-ray light curve in 1.5-12 kev from RXTE-ASM during the same period.

Table 1. HAGAR observations during the high state of activity.

\begin{tabular}{lcccc}
\hline \hline $\begin{array}{l}\text { Month } \\
2010\end{array}$ & $\begin{array}{c}\text { Total } \\
\text { duration } \\
(\mathrm{min})\end{array}$ & $\begin{array}{c}\text { Excess number } \\
\text { of on source } \\
\text { events }\end{array}$ & $\begin{array}{c}\text { Mean } \\
\gamma \text {-ray rate } \\
(\mathrm{min})\end{array}$ & $\begin{array}{c}\text { Signi- } \\
\text { ficance } \\
\sigma\end{array}$ \\
\hline February & 479 & 6418.22 & $13.4 \pm 1.05$ & 12.7 \\
March & 478 & 2524.23 & $5.3 \pm 1.1$ & 4.8 \\
April & 116 & 414 & $3.5 \pm 2.1$ & 1.7 \\
\hline
\end{tabular}

brightest flaring episodes was observed on February 17, 2010 (see Fig. 2), with the maximum flux between 6-7 Crab units. The source was detected with 5 sigma significance in less than 40 min of observations. We investigate in the following the multiwavelength behavior of Mrk 421 during the flaring episode.

\subsection{Flux variability during February 10-26, 2010}

The multiwavelength (radio to $\gamma$-rays) quasi-simultaneous light curve of Mrk 421 during February 10-26, 2010 based on observations in Sect. 3, is shown in Fig. 3. The top eight panels correspond to data from OVRO, SPOL, Swift-XRT, RXTEASM, RXTE-PCA, Swift-BAT, Fermi-LAT (0.2-2 GeV), and Fermi-LAT (2-300 GeV), respectively. The bottom panel corresponds to HAGAR data above $250 \mathrm{GeV}$.

A clear variation of flux over a period of seven days is observed in the optical, X-rays, and $\gamma$-rays during February 13-19, 2010. The peak flux in optical, X-rays, and low-energy $\gamma$-rays are observed to be around February 16, 2010 while in the VHE $\gamma$-ray band, the peak is seen on February 17, 2010 with a possible one-day lag compared to the situation at lower energies. The radio flux has not changed significantly during this time.

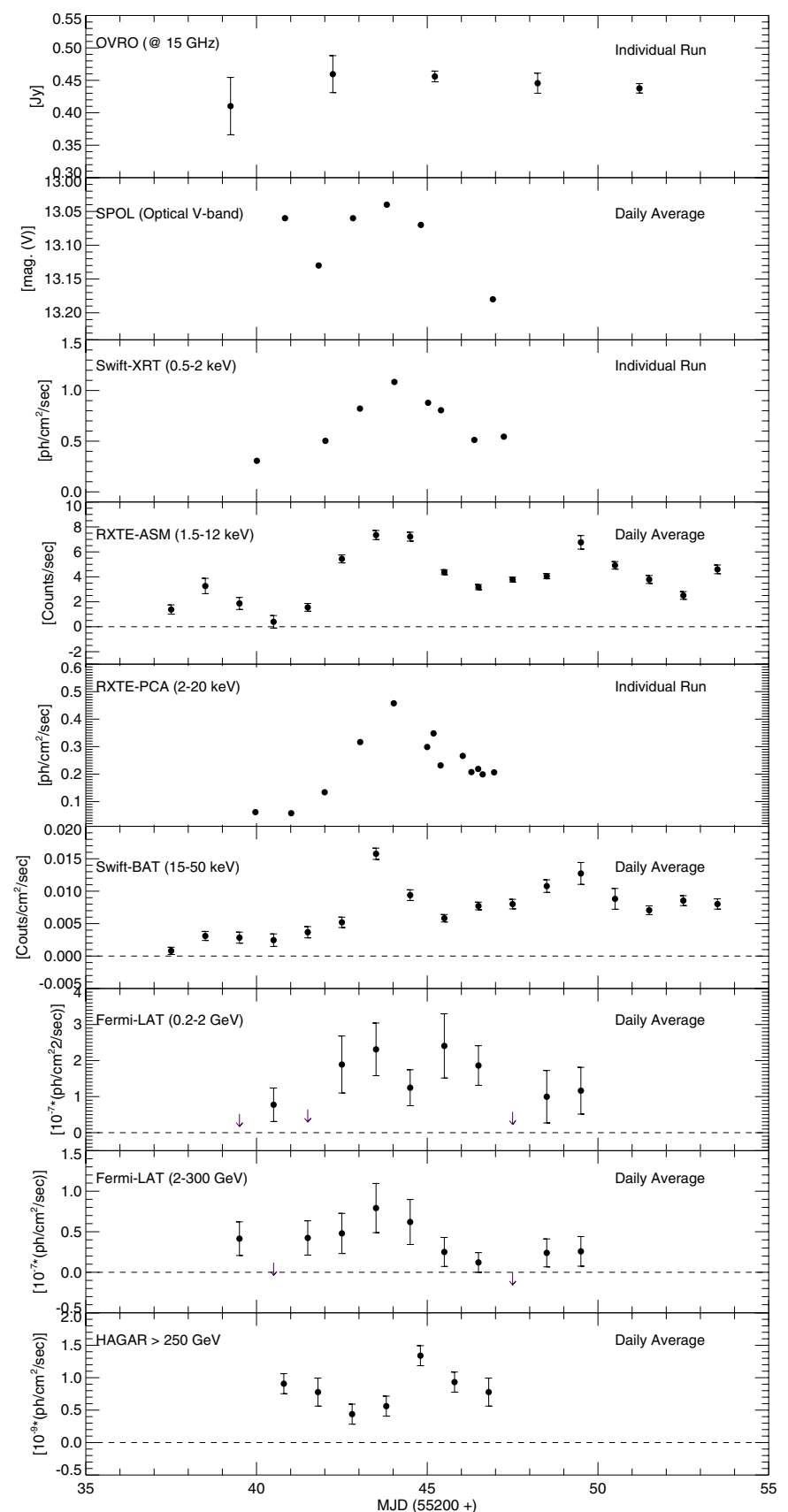

Fig. 3. Multiwavelength light curve of Mrk 421 during February 10-26, 2010.

\subsection{Intra-day and spectral variability during February 10-26,} 2010

Fermi-LAT data indicate an intra-day flux variability at energies $>200 \mathrm{MeV}$ during the TeV flare on February 17, 2010. An increase in the flux is seen in the first $9 \mathrm{~h}$ (MJD 55 244.0-55 244.4) of LAT observations, followed by a quiescence that lasts for a few hours (MJD 55 244.4-55 244.6) before the occurrence of another increase in flux during MJD 55244.7 to MJD 55 244.85. A similar trend was detected by the VERITAS collaboration (Ong 2010). HAGAR also observed a continuous decrease in the flux over a period of $\sim 2 \mathrm{~h}$ (MJD 55 244.8-55 244.86), which were simultaneous with LAT during the later part of the night (see Fig. 4). 


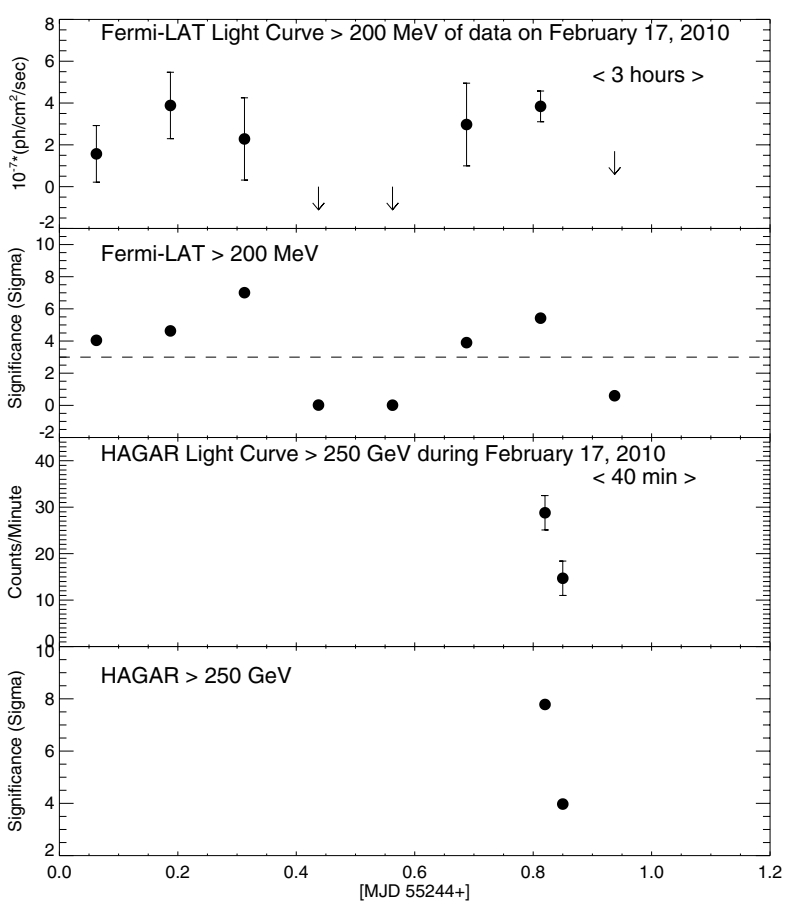

Fig. 4. Fermi-LAT and HAGAR intra-day light curve of Mrk 421 during February 17, 2010.

Table 2. Cutoff energy (PCA), spectral index (PCA) and photon in$\operatorname{dex}($ Fermi-LAT $>100 \mathrm{MeV})$ for four different activity states during February 13-19.

\begin{tabular}{lccc}
\hline \hline State & Cutoff PCA $(\mathrm{keV})$ & Index PCA & Index LAT \\
\hline Pre Flare & $29.1 \pm 6.3$ & $2.34 \pm 0.05$ & $1.65 \pm 0.03$ \\
Moderate Flare & $19.5 \pm 1.5$ & $1.91 \pm 0.03$ & $1.67 \pm 0.15$ \\
TeV Flare & $26.8 \pm 2.2$ & $2.03 \pm 0.02$ & $1.39 \pm 0.16$ \\
Post Flare & $35.2 \pm 2.3$ & $2.19 \pm 0.02$ & $2.06 \pm 0.04$ \\
\hline
\end{tabular}

Spectral variation was also detected over eleven days (February 12-22) of Fermi-LAT observations and seven days (February 13-19) of RXTE-PCA observations. A photon index of $1.39 \pm 0.17$, implying a flat/hard energy spectrum was observed in the Fermi-LAT data at energies above $100 \mathrm{MeV}$ on February 17, 2010. On the other hand, maximum hardening in RXTE-PCA data was observed on February 16 (Fig. 5 and Table 2). Spectral hardening in the X-rays (Tanihata et al. 2004) and $\gamma$-rays (Zweerink et al. 1997) during strong flares has been reported earlier also.

\section{Discussion}

Mrk 421 has shown several high states of flux activity in X-rays and $\gamma$-rays over the period of the past two decades (Buckley et al. 1996; Gaidos et al. 1996; Krennrich et al. 1999; Takahashi et al. 2000; Bhatt et al. 2002; Błażejowski et al. 2005; Bose et al. 2007; Giebels et al. 2007; Albert et al. 2007; Fossati et al. 2008; Horan et al. 2009; Vernetto \& Argo-Ybj Collaboration 2011; Binks 2010). It was observed in the brightest state of $\mathrm{TeV} \gamma$-ray flux during the years of 2000-2001, when its maximum flux reached as high as 14 Crab units above $1 \mathrm{TeV}$ (Tluczykont et al. 2010). It has also shown several mildly high states since 2001, but was not found to be in an extremely high state till 2010. Mrk 421

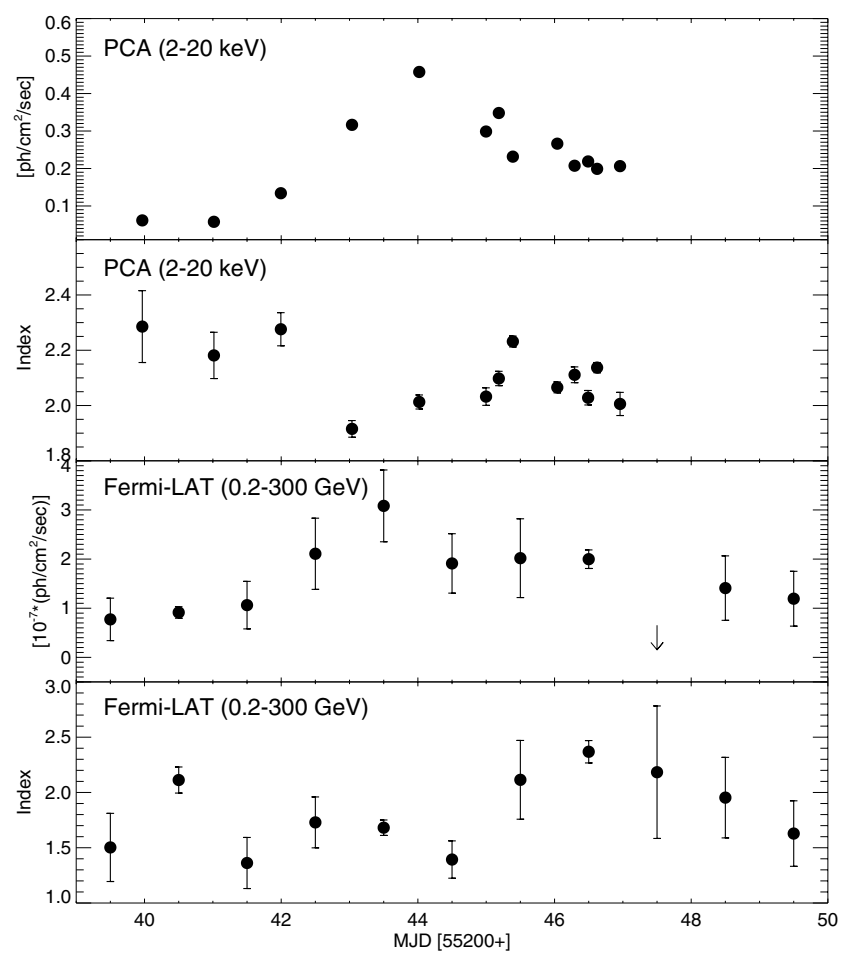

Fig. 5. RXTE-PCA light curve and spectral index are plotted in the first two panels, Fermi-LAT light curve in the range $0.2-300 \mathrm{GeV}$ and the photon index are plotted in bottom two panels.

had brightened up again in November 2009 and was observed by the VERITAS collaboration in an extremely high flux state on February 17, 2010. The VERITAS collaboration reported a maximum flux of 9 Crab units above $100 \mathrm{GeV}$ (Ong 2010) and the HESS collaboration (Tluczykont 2011) also found this source in a high state during their follow-up observations.

We have also detected this flare using the HAGAR telescope system, a few hours after the VERITAS observations, with a maximum flux of 6-7 Crab units above $250 \mathrm{GeV}$ (1 Crab unit $\sim 4.2 \gamma$-rays/minute above $250 \mathrm{GeV}$ ). HAGAR continued the observations of Mrk 421 over the next two months. The average flux during February, 2010 was found to be $\sim 3$ Crab units and $1 \mathrm{Crab}$ unit during March and April, 2010.

\subsection{Spectral energy distribution}

The SED of TeV blazars has a typical two-bump shape. It is often believed that the broadband emission from these sources is produced by a SSC mechanism. The lower energy bump, which peaks at infrared to X-ray is interpreted as synchrotron emission emitted by relativistic electrons gyrating in the magnetic field of the jet, and the $\mathrm{GeV} / \mathrm{TeV}$ bump is attributed to IC scattering of synchrotron photons by the same population of electrons that produces the synchrotron radiation.

A one-zone homogeneous SSC model developed by Krawczynski et al. (2004) was used to fit the multiwavelength data to obtain the SED. This model assumes a spherical blob of plasma of a comoving radius $R$, which travels with a bulk Lorentz factor $\Gamma$ toward the observer. The emission volume is filled with an isotropic population of electrons and a randomly oriented uniform magnetic field $B$. The energy spectrum of the injected electrons in the jet frame is described by a broken power law with low-energy $\left(E_{\min }\right.$ to $\left.E_{\mathrm{b}}\right)$ and high-energy $\left(E_{\mathrm{b}}\right.$ to $\left.E_{\max }\right)$ 
components with indices of $p 1$ and $p 2$. The emitted radiation is Doppler-boosted by the Doppler factor

$\delta=[\Gamma(1-\beta \cos (\theta))]^{-1}$,

where $\beta$ is the bulk velocity of the plasma in units of the speed of light and $\theta$ the angle between jet axis and the line of sight in the observer frame. The VHE $\gamma$-ray spectrum is corrected for absorption by the extragalactic background light (Franceschini et al. 2008).

The radius of the emission zone is constrained by the variability time scales. Variability present in X-ray and $\gamma$-ray data is on the order of one day, so we have chosen $t_{\mathrm{VAR}} \sim 1$ day. The comoving radius of the emission zone is defined as

$R \sim c \delta t_{\mathrm{var}} /(1+z)$,

we attempted to obtain SEDs for different flux states using multiwavelength data of the Mrk 421 during HAGAR observations of February 13-19, 2010 in an attempt to search for any changes in the physical parameters over this period. This multiwavelength data is divided into four states according to the flux state of the source as follows: State 1: pre flare (13-15 February), State 2: moderate flare (16 February), State 3: TeV flare (17 February), and State 4: post flare (18-19 February). The pre flare state of the source is a moderately high state.

The Fermi-LAT data were divided into three bins (0.1-1 GeV, 1-3 GeV and 3-300 GeV) to obtain the spectrum of Mrk 421 for "State 1", "State 2" and "State 4" by freezing the photon index to $1.65,1.67$ and 2.06 respectively. These photon indices were obtained by analyzing the $0.1-300 \mathrm{GeV}$ data from Fermi-LAT. The Fermi-LAT spectrum of February 17, 2010 was obtained by dividing Fermi-LAT data into four bins $(0.1-1 \mathrm{GeV}$, 1-3 GeV, 3-10 GeV and 10-300 GeV) by freezing the photon index to 1.39 , obtained by the analysis of the $0.1-300 \mathrm{GeV}$ data. The best-fit photon indices and cutoff energies for all four states are presented in Table 2.

The magnetic field, Doppler factor $(\Gamma \sim \delta)$, electron energy density, break present in electron injection spectrum $\left(E_{\mathrm{b}}\right), p 1$, $p 2$, minimum and maximum electron energies were used as free parameters while fitting the model to optical, X-ray, and $\gamma$-ray data. The angle between the jet axis and the line of sight in the observer frame was taken to be $2.6^{\circ}$.

\subsection{Evolution of the SED during the high state}

The Fermi-LAT collaboration (Abdo et al. 2009) had reported the photon index of the energy spectrum of Mrk 421 to be 1.78 from the first 5.5 months of their observations. This refers to an average spectrum mostly during the quiescent state. However, during "State 1" (see Fig. 6a), we find the energy spectrum to be flatter with a photon index of 1.65 corresponding to 0.1-300 GeV Fermi-LAT data. Also, we see a plateau in the light curves during this state in the Fermi-LAT data (in 2-300 GeV energy bands) and 15-50 keV Swift-BAT data (see Fig. 3). The presence of a plateau in the light curve indicates that the injection time scales of energetic electrons are longer than the cooling time scales, and the entire volume of emission zone is radiating. Plateaux are also observed in other $\gamma$-ray sources like $3 c 454.3$ (Abdo et al. 2011a).

Flares in optical, X-ray, low-energy $(<2 \mathrm{GeV}) \gamma$-ray, and HE (2-300 GeV) $\gamma$-ray band were seen in "State 2 ". The corresponding SED is shown in Fig. 6b. These low and HE $\gamma$-ray flares could be caused by upscattering of X-ray photons by lowenergy electrons that are produced by SSC cooling in "State 1".
As an effect of the low-energy flare, the low-energy part of SED in "State 2" is much flatter than that in "State 1".

The observed $\mathrm{GeV} / \mathrm{TeV}$ flares above $250 \mathrm{GeV}$ on 17 February could be produced by upscattering of X-ray photons in Swift-BAT energy range by higher energy electrons (see Fig. $6 c$ for the SED). The presence of a passing shock might have accelerated the electrons to higher energies in the emission region.

The source is brighter at lower energy $\gamma$-ray in "State 4", which could be due to cooling of electrons after the high-energy flare. The corresponding SED is shown in the Fig. 6d.

The derived SED parameters for each state are listed in Table 4, and in Fig. 6e we illustrate the change that the SED undergoes during the four different states of the source.

\subsection{Light curve}

Significant flux variation in optical, X-ray, and $\gamma$-ray bands is detected during February, 2010. The highest energy tail of the electron energy distribution $\left(\gamma \geq \gamma_{\mathrm{br}}\right)$ is responsible for the production of the observed X-ray synchrotron continuum at $\geq 0.5 \mathrm{keV}$, while the $\mathrm{TeV} \gamma$-rays might be produced through upscattering of synchrotron photons by the same population of electrons. The observed optical, X-ray variability during February, 2010 may be explained by injection of fresh electron in emission zone and cooling of the electrons due to SSC mechanism. The Swift-BAT light curve showed faster variability than RXTE-ASM, which could be due to the cooling effect of highenergy electrons, which produce $\mathrm{X}$-rays at the $15-50 \mathrm{keV}$ range.

The observed $\gamma$-ray variability is mainly divided into two bands, $<2 \mathrm{GeV}$ and above $2 \mathrm{GeV}$. The $0.2-2 \mathrm{GeV} \gamma$-rays observed by Fermi-LAT could be produced by low-energy electrons through IC scattering of UV synchrotron photons. Fermi-LAT detected a significant variation in flux in the $0.2-2 \mathrm{GeV}$ band, observed over a period of eleven days during February 12-22, 2010.

The observed HE ( $>2 \mathrm{GeV}) \gamma$-rays by Fermi-LAT and VHE $\gamma$-rays by HAGAR could be produced by IC scattering of the electrons having a Lorentz factor in the range $\sim 10^{4}-10^{5}$. The observations from Fermi-LAT $(2-300 \mathrm{GeV})$ show a symmetrical flare centered around February 16, 2010 but VHE $\gamma$-rays flux, observed by HAGAR reaches peak with a one-day lag on February 17, 2010. A fresh injection of high-energy electrons accelerated by shock could be responsible for the observed flare in $\gamma$-rays. If the SSC cooling time scale is shorter than the lightcrossing time of the emission zone, the flare could be symmetric.

\subsection{Cross-correlation study and time lag}

From the X-ray and $\gamma$-ray light curves (Figs. 2 and 3), we can see that fluxes of Mrk 421 in these bands are roughly correlated. We investigated this correlation between X-ray and $\gamma$-ray bands using the cross-correlation function $(\mathrm{CCF})$ and the $z$-transformed discrete correlation function (ZCDF) (Alexander 1997), a freely available FORTRAN 77 ZDCF code ${ }^{6}$. Observed lag and correlation coefficient between RXTE-ASM, Swift-BAT and HAGAR using CCF and ZDCF algorithms are listed in Table 3 for various cases. The time lag between X-ray and $\gamma$-ray emissions might impose constraints on emission region and could distinguish between SSC and external Compton (EC) models.

${ }^{6}$ http://www. weizmann.ac.il/home/tal/zdcf2.html 
A. Shukla et al.: Multiwavelength study of the TeV blazar Mrk 421 during a giant flare

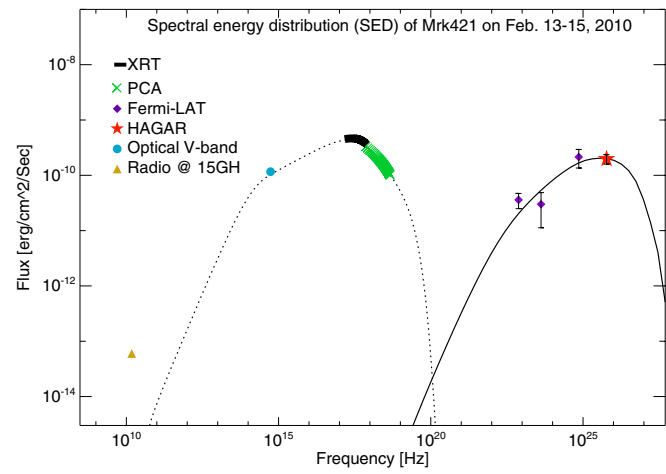

(a)

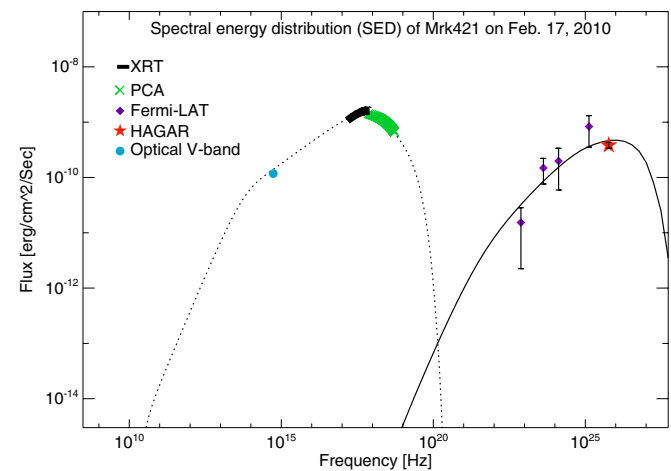

(c)

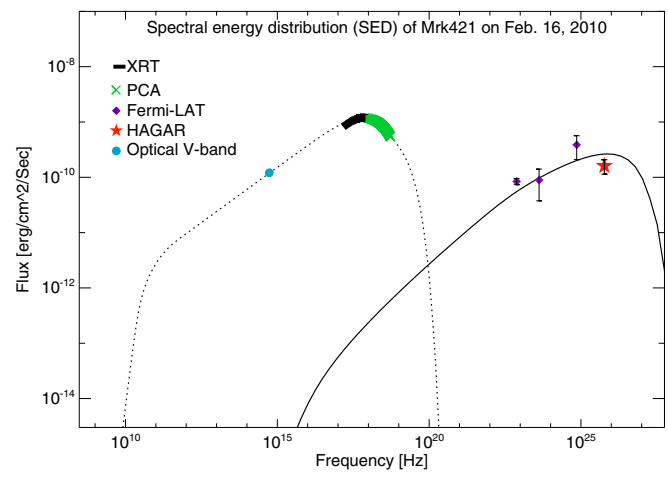

(b)



(d)

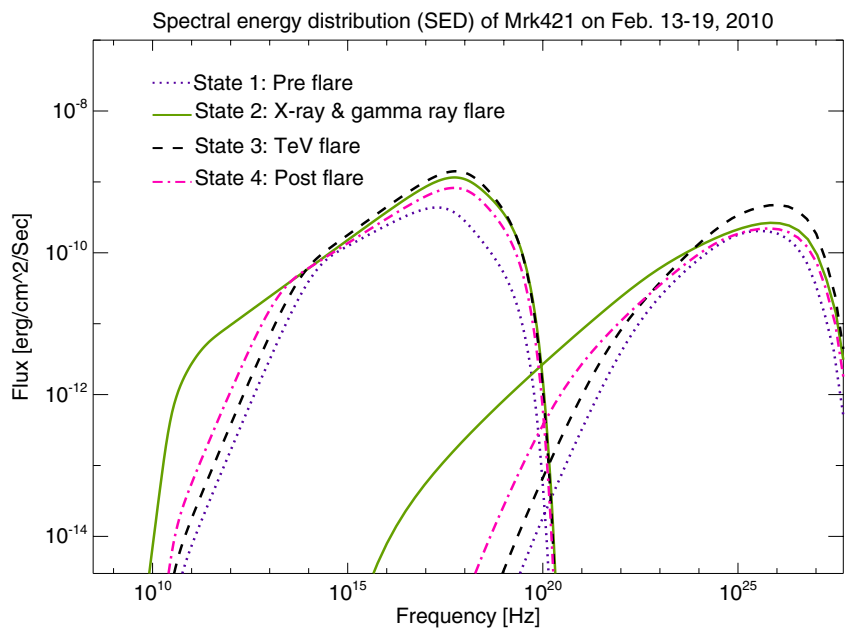

(e)

Fig. 6. SEDs of Mrk 421 during February 2010.

Table 3. Correlation coefficient and time lag of Mrk 421 emission during February-April, 2010.

\begin{tabular}{lcccc}
\hline \hline Instruments & Lag (Days) & $\begin{array}{c}\text { Correlation } \\
\text { coefficient }\end{array}$ & Algorithm & $\begin{array}{c}\text { Data used } \\
2010\end{array}$ \\
\hline RXTE-ASM vs. Swift-BAT & 0.0 & 0.81 & CCF & $13-19$ February \\
RXTE-ASM vs. Swift-BAT & 0.0 & 0.77 & ZDCF & $10-27$ February \\
RXTE-ASM vs. HAGAR & 1.3 & 0.62 & ZDCF & February to April \\
Swift-BAT vs. HAGAR & 1.3 & 0.88 & CCF & $13-19$ February \\
Swift-BAT vs. HAGAR & 1.3 & 0.82 & ZDCF & $13-19$ February \\
Swift-BAT vs. HAGAR & 1.3 & 0.74 & ZDCF & February to April \\
\hline
\end{tabular}


Table 4. SED parameters obtained by fitting to data using $t_{\mathrm{var}} \sim 1$ day.

\begin{tabular}{|c|c|c|c|c|c|c|c|c|c|c|c|}
\hline $\begin{array}{l}\text { State } \\
- \\
-\end{array}$ & $\begin{array}{c}\text { Magnetic } \\
\text { field } \\
(\mathrm{G})\end{array}$ & $\begin{array}{l}\text { Doppler } \\
\text { factor } \\
(\delta)\end{array}$ & $\begin{array}{c}\log E_{\min }{ }^{a} \\
{[\mathrm{eV}]} \\
-\end{array}$ & $\begin{array}{c}\log E_{\max } b \\
{[\mathrm{eV}]} \\
-\end{array}$ & $\begin{array}{c}E_{\text {break }}^{c} \\
{[\mathrm{eV}]} \\
-\end{array}$ & $p 1$ & $\begin{array}{c}p 2 \\
-\end{array}$ & $\begin{array}{c}\mathrm{Sy}_{\mathrm{pk}}{ }^{d} \\
{\left[10^{17}\right]} \\
(\mathrm{Hz})\end{array}$ & $\begin{array}{c}\mathrm{IC}_{\mathrm{pk}} e \\
{\left[10^{25}\right]} \\
(\mathrm{Hz})\end{array}$ & $\begin{array}{c}U_{\mathrm{e}}^{f} \\
{\left[10^{-3}\right]} \\
(\mathrm{erg} / \mathrm{cc})\end{array}$ & $\begin{array}{c}\eta^{g} \\
{\left[u_{\mathrm{e}}^{\prime} / u_{B}^{\prime}\right]} \\
-\end{array}$ \\
\hline State1 & 0.026 & 19.5 & 9.6 & 12.1 & 11.3 & 2.4 & 4.3 & 1.93 & 3.58 & 0.9 & 33.46 \\
\hline State & 29 & 22.0 & 8.0 & 12.1 & 11.40 & 2.2 & 3.9 & 5.74 & 7.02 & 1.4 & 41.83 \\
\hline State3 & 0.029 & 21.0 & 9.4 & 12.1 & 11.45 & 2.2 & 4.1 & 6.13 & 11.53 & 1.0 & 29.88 \\
\hline State4 & 0.028 & 21.0 & 9.1 & 12.1 & 11.45 & 2.3 & 4.1 & 5.53 & 6.76 & 8.5 & 27.24 \\
\hline
\end{tabular}

(a) $E_{\min }$ : minimum value of energy of the electrons present in the emission zone. ${ }^{(b)} E_{\max }$ : maximum value of energy of the electron present in the emission zone. ${ }^{(c)} E_{\text {break }}$ : Break in the electron injection spectrum. ${ }^{(d)} \mathrm{Sy}_{\mathrm{pk}}$ : peak value of synchrotron bump. ${ }^{(e)} \mathrm{IC}_{\mathrm{pk}}$ : peak value of $\mathrm{IC}$ bump. ${ }^{(f)} \mathrm{U}_{\mathrm{e}}$ : electron energy density. ${ }^{(g)} \eta$ : equipartition coefficient.

\section{Conclusions}

We found satisfactory fits for all four states with the one-zone SSC model described in Sect. 5.1. The observed pre-to post-flare evolution of the SED and light curve are explained by the model. Changes in the physical conditions such as Doppler factor and magnetic field during these observations are indicated by modeling of the multiwavelength data. It appears that changes in these parameters are related to the activity of the source. The change in jet flow from "State 1" to "State 2" may lead to a shock in the jet. We also found small changes in the particle energy density and the magnetic field strength from "State 1" to "State 2". The best fits obtained by fitting multiwavelength data to the SSC model during the rising part of the flare ("State 2" and "State 3") are found for a power law index of the electron injection spectrum before break of $p 1=2.2$. Because $p 1=2.2$ is a result of the Fermi first-order mechanism, it strengthens the belief that a strong shock might have accelerated the electrons in the emission zone and caused the flare by SSC mechanism. Very recently, Abdo et al. (2011b) showed that electrons present in the emission zone of Mrk 421 might be accelerated by a Fermi first-order mechanism at the shock front. The best-fit parameters from the modeling of the SED using multiwavelength data for "State 1" indicate that an aged population of electrons with $p 1=2.4$ was present in the emission zone. The light curve during the same period suggests that the entire volume of the emission zone was radiating. The observed change in the electron injection spectrum $p 1=2.3$ during the decaying part of the flare might be considered to be a result of SSC cooling mechanism.

The observed break in the electron injection spectrum could also be explained as a cooling break, where the escape time of an electron of Lorentz factor $\gamma_{\mathrm{c}}$, equals the radiative cooling time. It is observed from our results that the cooling break changes with the state of the source. We have also found that both SED peaks move toward higher energy as the source flux increases, and return to lower energies as it decreases. Spectral hardening is also observed at X-rays and $\gamma$-rays at the time of the flare (high state) (see Fig. 5, Table 2). A departure from equipartition is also observed during this high state. The emission process at the time of high activity is more complex than in the quiescent state. Energy-dependent variability observed from Fermi-LAT has shown that the source is much more complex than anticipated. The emission from Mrk 421 also showed an intra-day variability on February 17, 2010, in $\gamma$-ray bands above $0.2 \mathrm{GeV}$ in Fermi-LAT data and $>250 \mathrm{GeV}$ from HAGAR data. We could not significantly detect this sub-hour-scale variability from Fermi-LAT owing to the large error bars. The detection of sub-hour variability from Fermi-LAT and HAGAR could constrain the size of the emission region. The present results of correlation studies between X-ray and $\gamma$-ray flux variability are not conclusive enough to distinguish between the one-zone SSC or EC model. However, the observed hardening of the emission spectra obtained from Fermi-LAT and RXTE-PCA data with a one-day lag (see Fig. 5 and Table 2) is inconsistent with the SSC model and opens up the possibility of multizone-SSC or EC models.

\section{Summary}

i. The HAGAR Cherenkov telescope has detected VHE $\gamma$-rays from the TeV blazar Mrk 421 during 2010 February to April, with the blazar being in a high state of activity above $250 \mathrm{GeV}$. The emission reached a peak on February 17, with a maximum flux of $\sim 7 \mathrm{Crab}$ units, indicating a flare.

ii. An energy-dependent variation of flux in the HE $\gamma$-rays $(0.1-300 \mathrm{GeV})$ has been observed by Fermi-LAT during the flare in February, 2010.

iii. Multiwavelength data indicate a variation in the spectral index during the high state.

iv. A weak correlation between X-rays and VHE $\gamma$-ray has been found with a time lag of $\sim 1$ day.

v. The observed multiwavelength SEDs during February 1319, 2010 indicate changes in the physical conditions such as magnetic field, Doppler factor, and particle energies in the emission zone. The multiwavelength flare during February 16-17, 2010 and changes in physical conditions of the emission zone are explained as an effect of a passing shock in the jet.

Acknowledgements. This work used results provided by the ASM/RXTE teams at MIT. This study also used Swift/BAT transient monitor results provided by the Swift/BAT team. This research has also made use of data obtained from the High Energy Astrophysics Science Archive Research Center (HEASARC), provided by NASA's Goddard Space Flight Center. Data from the Steward Observatory spectropolarimetric monitoring project were used. This program is supported by Fermi Guest Investigator grants NNX08AW56G and NNX09AU10G. Radio data at $15 \mathrm{Ghz}$ is used from OVRO $40 \mathrm{M}$ Telescope and this Fermi blazar monitoring program is supported by NASA under award NNX08AW31G, and by the NSF under award 0808050 . We are grateful to the engineering and technical staff of IIA and TIFR, who have taken part in the construction of the HAGAR telescopes and contributed to the setting-up of the front-end electronics and the data acquisition. We are grateful to R. Cowsik and B. V. Sreekantan for their keen interest and encouragement in the development of the HAGAR facility. We also thank the anonymous referee for his/her suggestions, which improved the manuscript.

\section{References}

Abdo, A. A., Ackermann, M., Ajello, M., et al. 2009, ApJ, 707, 1310 Abdo, A. A., Ackermann, M., Ajello, M., et al. 2011a, ApJ, 733, L26 Abdo, A. A., Ackermann, M., Ajello, M., et al. 2011b, ApJ, 736, 131 Aharonian, F. A. 2000, New Astron., 5, 377

Aharonian, F. A., Akhperjanian, A. G., Andronache, M., et al. 1999, A\&A, 350, 757

Albert, J., Aliu, E., Anderhub, H., et al. 2007, ApJ, 663, 125

Aleksić, J., Anderhub, H., Antonelli, L. A., et al. 2010, A\&A, 519, A32 
Alexander, T. 1997, in Astronomical Time Series, ed. D. Maoz, A. Sternberg, \& E. M. Leibowitz, Astrophysics and Space Science Library, 218, 163 Atwood, W. B., Abdo, A. A., Ackermann, M., et al. 2009, ApJ, 697, 1071 Bhatt, N., Agarwal, N. K., Bhat, C. K., et al. 2002, BASI, 30, 385 Binks, J. 2010, APS Four Corners Section Meeting Abstracts, L1004 Błażejowski, M., Blaylock, G., Bond, I. H., et al. 2005, ApJ, 630, 130 Bose, D., Chitnis, V. R., Vishwanath, P. R., et al. 2007, Ap\&SS, 309, 111 Bradt, H. V., Rothschild, R. E., \& Swank, J. H. 1993, A\&AS, 97, 355 Buckley, J. H., Akerlof, C. W., Biller, S., et al. 1996, ApJ, 472, L9

Burrows, D. N., Hill, J. E., Nousek, J. A., et al. 2005, Space Sci. Rev., 120, 165 Cash, W. 1979, ApJ, 228, 939

Chitnis, V. R., Pendharkar, J. K., Bose, D., et al. 2009, ApJ, 698, 1207

Cowsik, R., Bhat, P. N., Chitnis, V. R., Acharya, B. S., \& Vishwanath, P. R. 2001, in Int. Cosmic Ray Conf., 7, 2769

Dermer, C. D., \& Schlickeiser, R. 1993, ApJ, 416, 458

Di Sciascio, G., \& ARGO-YBJ Collaboration. 2010, Mem. Soc. Astron. Ital., 81,326

Dickey, J. M., \& Lockman, F. J. 1990, ARA\&A, 28, 215

Fossati, G., Buckley, J. H., Bond, I. H., et al. 2008, ApJ, 677, 906

Franceschini, A., Rodighiero, G., \& Vaccari, M. 2008, A\&A, 487, 837

Gaidos, J. A., Akerlof, C. W., Biller, S., et al. 1996, Nature, 383, 319

Ghisellini, G., \& Madau, P. 1996, MNRAS, 280, 67

Giebels, B., Dubus, G., \& Khélifi, B. 2007, A\&A, 462, 29

Heck, D., Knapp, J., Capdevielle, J. N., Schatz, G., \& Thouw, T. 1998, CORSIKA: a Monte Carlo code to simulate extensive air showers
Horan, D., Acciari, V. A., Bradbury, S. M., et al. 2009, ApJ, 695, 596 Isobe, N., Sugimori, K., Kawai, N., et al. 2010, PASJ, 62, L55

Krawczynski, H. 2004, New Astron. Rev., 48, 367

Krawczynski, H., Hughes, S. B., Horan, D., et al. 2004, ApJ, 601, 151

Krennrich, F., Biller, S. D., Bond, I. H., et al. 1999, ApJ, 511, 149

Mannheim, K. 1998, Science, 279, 684

Mattox, J. R., Bertsch, D. L., Chiang, J., et al. 1996, ApJ, 461, 396

Mücke, A., Protheroe, R. J., Engel, R., Rachen, J. P., \& Stanev, T. 2003, Astropart. Phys., 18, 593

Ong, R. A. 2010, The Astronomer's Telegram, 2443, 1

Petry, D., Bradbury, S. M., Konopelko, A., et al. 1996, A\&A, 311, L13

Punch, M., Akerlof, C. W., Cawley, M. F., et al. 1992, Nature, 358, 477

Richards, J. L., Max-Moerbeck, W., Pavlidou, V., et al. 2011, ApJS, 194, 29

Saha, L., Acharya, B. S., Anupama, G. C., et al. 2011, in International Cosmic Ray Conference., id:OG2.5,1129

Smith, P. S., Montiel, E., Rightley, S., et al. 2009 [arXiv: 0912 . 3621]

Takahashi, T., Kataoka, J., Madejski, G., et al. 2000, ApJ, 542, L105

Tanihata, C., Kataoka, J., Takahashi, T., \& Madejski, G. M. 2004, ApJ, 601, 759

Tluczykont, M. 2011 [arXiv: 1106. 1035]

Tluczykont, M., Bernardini, E., Satalecka, K., et al. 2010, A\&A, 524, A48

Vernetto, S., \& Argo-Ybj Collaboration 2011, Astrophys. Space Sci. Trans., 7, 65

Yadav, K. K., Chandra, P., Tickoo, A. K., et al. 2007, Astropart. Phys., 27, 447

Zweerink, J. A., Akerlof, C. W., Biller, S. D., et al. 1997, ApJ, 490, L141 